\title{
A Contrastive Study of Master Thesis Acknowledgements by Taiwanese and North American Students
}

\author{
Stephanie W. Cheng \\ Graduate Institute of TESOL, National Chiao Tung University, Hsinchu, Chinese Taipei \\ Email: scheng@mail.nctu.edu.tw
}

Received January $2^{\text {nd }}, 2012$; revised February $17^{\text {th }}, 2012$; accepted February $26^{\text {th }}, 2012$

\begin{abstract}
Thesis acknowledgements are a written part genre in which graduate students express their gratitude towards a number of addressees upon completion of theses. Previous studies on thesis acknowledgements have focused on the expressions of gratitude or their generic structure. However, socio-cultural values and norms can shape the ways people express thanks and influence the rhetorical structure of a genre like thesis acknowledgements. The present study compares and contrasts the use of thanking strategies in 60 thesis acknowledgements written in English by Taiwanese and North American master students. Results show that Taiwanese students (TS) use more thanking strategies than North American students (NAS); specifically, they employ more explicit thanking strategies but less implicit thanking strategies than NAS. They also use more complex thanking strategies but less simple thanking strategies than NAS. Interestingly, the two groups vary in the arrangement of addressees and the choice of strategies for various addressees, reflecting different cultural perceptions of expressing gratitude. For example, NAS appear to make a more flexible arrangement of advisors and family members than TS. These subtle differences between TS and NAS thesis acknowledgements reveal Taiwanese and North American students' embedded socio-pragmatic perceptions of writing this genre.
\end{abstract}

Keywords: Acknowledgements; Thanking Strategies; Expressions of Gratitude; Genre

\section{Introduction}

Research in English for Academic Purposes (EAP) has received growing attention and produced studies of various academic text types in different settings. Acknowledgements appear widely in academic writing, especially in theses and dissertations. Thesis acknowledgements provide student writers a chance to express their gratitude for the intellectual guidance, personal assistance as well as encouragement and moral support they have received in completing their research. Acknowledgements in theses and dissertations, thus, signify not only a unique rhetorical act for interpersonal relationship, but also a reflection of student writers' socio-cultural values and identity in academia. However, the acknowledgement section has received much less attention than the other sections in theses and dissertations, namely, Abstract, Introductions, Literature review, Methods, Results, Discussion and Conclusion.

While acknowledgements encourage students to express their gratitude explicitly and honestly and to show their interpersonal network in the academic community, students may have problems adequately expressing gratitude in this academic discourse (Hyland, 2004). Specifically, writing thesis acknowledgements involves social and cultural pragmatism. Many questions may arise: how do graduates in different social contexts and cultures perceive and express gratitude in thesis acknowledgements? Are there socio-cultural preferences in arranging the thanked addressees in thesis acknowledgements? Are there socio-cultural variations in the use of thanking strategies and their linguistic realizations?

Previous studies on thesis acknowledgements have mainly focused on the expressions of gratitude and their generic struc- ture (Hyland, 2004; Hyland \& Tse, 2004). Very few studies have compared thesis acknowledgements by native and nonnative students of English. Furthermore, existing research on acknowledgements has rarely taken a socio-cultural perspective in analyzing how thanks are expressed, who are thanked, and how and why the thanked addressees are arranged in thesis acknowledgements.

Therefore, this study attempts to compare and contrast master thesis acknowledgements by native and non-native speakers of English, specifically, North American and Taiwanese students, in terms of thanking strategies, linguistic realizations of thanking strategies, relations between thanking strategies and the thanked addressees, and arrangement preference for the thanked addressees.

\section{Approaches to Acknowledgements in Academic Texts}

Over the course of the last century, acknowledgements have become widespread and an important feature of scholarly texts (Bazerman, 1984, 1988). From the early times, acknowledgements in academic research has been of great interest to bibliometricians who use quantitative methods in seeking to trace the lines of research networks and relationships amongst academic journals (Cronin, 1991, 1995; Cronin, Mckenzie \& Rubio, 1993; Cronin, Mckenzie, \& Stiffler, 1992; Cronin \& Overfelt, 1994). Although these researchers classify acknowledgements according to their features and functions, they have not examined the organizational patterns and linguistic realizations of acknowledgements in any particular genre.

More recently, studies have taken a genre analysis approach 
to investigate the rhetorical structure and linguistic realizations of acknowledgements in research articles and theses and dissertations (Al-Ali, 2010; Giannoni, 2002; Hyland, 2003, 2004; Hyland \& Tse, 2004). From a genre-analytic perspective, for example, Giannoni (2002) examined the socio-pragmatic construction and textualization of acknowledgement texts in English and Italian research articles, and indicated that English and Italian writers have their rhetorical preferences of acknowledgements based on issues such as generic complexity and staging, personal involvement and peer-reference, authorial responsibility and pragmatic appropriacy. It was also found that Italian writers employ a larger number of impersonal constructions, while English writers had a wider range and slightly higher proportion of overt thanking expressions. This finding implies that some thanking expressions may be culture-specific. Different cultural norms and social expectations probably play a role in determining the choices of expressions of gratitude (Bach \& Harnish, 1979). Hyland (2003, 2004) and Hyland \& Tse (2004) investigated English Master thesis and PhD dissertation acknowledgements by non-native speakers of English in Hong Kong and proposed a move structure framework, including three moves: 1) Reflecting Move; 2) Thanking Move, which includes four steps: presenting participants, thanking for academic assistance, thanking for resources, and thanking for moral support; and 3) Announcing Move, including 2 steps: accepting responsibility and dedicating the thesis. Adding to Hyland's (2004) three-move framework, Al-Ali (2010) proposed an eight-move structure to incorporate the socio-cultural components (e.g., religious beliefs, academic and social conventions) of the Arabic acknowledgement practice. For instance, some specific features in Arabic acknowledgements are praising and thanking Allah (God) and invoking and blessing from God's (Allah's) favors upon the thanked addressees.

A move analysis identifies the rhetorical structure of a certain genre in relation to the communicative purposes of the genre, whereas a pragmatic analysis focuses more on the strategy use of speakers/writers and analyzes the meaning of words and sentences on the basis of context, the inferred intent of speakers/writers, and the status and relationships of those involved. As mentioned earlier, previous studies have conducted move analysis to analyze the rhetorical structure of thesis acknowledgements. However, a pragmatic analysis, in particular, a comparison of thesis acknowledgements by two groups of student writers from different cultures, can further reveal the students' embedded socio-pragmatic perceptions of writing this genre.

From a pragmatic perspective, acknowledgements are written forms of the speech act of thanking. Searle (1969) defined thanking as an expressive illocutionary act; that is, when thanking, the speaker expresses gratitude for the hearer's participation in a prior action that is beneficial to the speaker. The speaker feels grateful or appreciative for the prior action, and makes an utterance that counts as an expression of gratitude or appreciation. Similarly, the main communicative purpose in thesis and dissertation acknowledgements is for student writers to show appreciation and indebtedness to the assistance and contributions received from others to accomplish their academic research. Bach and Harnish (1979) indicated that the genuine feeling of gratitude is not necessary, but the expressions of gratitude should meet social expectations. Al-Ali (2010) also found that Arabic doctoral students tend to use certain preferred address forms, and social honorifics together with various gratitude expressions to respond to different types of the academic and social community members. It is clear that socio-cultural values and norms play a role in shaping this genre. Additionally, a unique feature of thesis and dissertation acknowledgements that is different from thanking in everyday conversation is the multitude of thanked addressees. The relationships between student writers and various addressees in light of their respective assistance and support to the students may affect how they are thanked or how they are arranged in acknowledgements. Therefore, in addition to analyzing the use of thanking strategies, the present study takes a step further to examine the arrangement of the thanked addressees and the relations between thanking strategies and addressees in thesis acknowledgements, which have not yet been investigated.

\section{Methodology}

\section{Corpus}

The study is based on 60 MA thesis acknowledgement sections written in English by non-native and native speakers of English, namely, Taiwanese students (TS) and North American students (NAS). Each of the two corpora (TS and NAS) consists of 30 acknowledgements published in the recent five years (2005-2009) from the discipline of applied linguistics. The selection of this single discipline was to minimize the possible influence of disciplinary conventions in expressing gratitude in acknowledgements.

Both TS and NAS data were collected from online databases of dissertations and theses in Taiwan and in the United States, namely, National Digital Library of Theses and Dissertations in Taiwan, and ProQuest Dissertations and Theses Database in the United States. Some criteria were involved to carefully examine the native identities of the thesis authors for proper representation of Taiwanese students or American/Canada students, such as the origins of the thesis writers' names, their resumes, or the explicit indication of their background or culture. In the examples taken from the corpora, the names of people or institutions or anything that revealed the identity of people involved were replaced by pseudo names. The TS corpus consists of a total of 9481 running words, whereas the NAS corpus consists of 7293 words. The length of the acknowledgements ranges from 188 to 598 words, with an average of 316 words for TS corpus; however, in the NAS corpus, the length of the texts ranges from 35 to 640 words, with an average of 243 words.

\section{Coding Scheme and Procedures of Data Analysis}

The acknowledgements were analyzed for their pragmatic patterns of expressions, in terms of thanking strategies and linguistic realizations, to determine how student writers express thanks. A two-level scheme was developed for the analysis of thanking strategies. At the lower level, sentences in each acknowledgement text was segmented into semantic categories. Seven types of semantic categories were identified: 1) thanking, explicitly using words that express gratitude, such as thank, appreciate, gratitude, indebted, and grateful; 2) addressees; 3) reasons due to the favor received, such as insightful comments, efforts, and academic guidance; 4) reasons due to positive feelings, such as love, support, and encouragement; 5) reasons due to a mixture of favor and positive feelings; 6) indispensability, using double negation structure, such as without... not... and were it not... not..., and 7) elaboration, elaborating after ex- 
pressing thanks once. For example, following are sentences expressing gratitude towards the thesis advisor in one of the acknowledgements in the TS corpus:

1) I would like to express my profound gratitude to Dr. WenPing Huang, my thesis advisor, for her constant guidance and patience with this thesis. Dr. Huang's careful reading and insightful suggestions on all the drafts have made the thesis more complete (TS10).

The sentences can be divided into 4 units, each of which falls into a corresponding semantic category in the scheme:

a) [thanking] I would like to express my profound gratitude $-->$

b) [addressee] to Dr. Wen-Ping Huang, my thesis advisor, $-->$

c) [reason (favor)] for her constant guidance and patience with this thesis. -->

d) [elaboration] Dr. Huang's careful reading and insightful suggestions on all the drafts have made the thesis more complete.

At the higher level, different semantic categories were then combined to form various thanking strategies. As we know, the essential elements of thanking are thanking and addressee towards whom thanking is directed. However, thanking can be explicit, using overt thanking words or phrases, or implicit, expressing gratitude without using overt thanking words or phrases. Therefore, a thanking strategy consists of the semantic category of addressees with or without an overt thanking word/ phrase and other semantic categories. In other words, the semantic category of addressee(s) is an obligatory category in a thanking strategy, while the other semantic categories are optional. In the above example, the four semantic categories form a thanking strategy which can be represented as:

[thanking] + [addressees] + [reason (favor) $]+$ [elaboration]

Thanking strategies with an overt thanking word (i.e., with the [thanking] category) were termed explicit thanking strategies; on the contrary, those without an overt thanking word were termed implicit thanking strategies. Depending on the complexity of the semantic categories in a thanking strategy, explicit thanking strategies were divided into simple thanking strategies and complex thanking strategies. A simple thanking strategy consists of one overt thanking word, one or more addressees (as a group), together with or without another semantic category, while a complex thanking strategy consists of one or more overt thanking words and addressees, together with more than one of the other semantic categories. Example 1 from above was classified as $\mathrm{C}$, one of the complex thanking strategies. Representations of all thanking strategies are shown in Table 1.

In acknowledgement texts, it is possible that student writers use more than one sentence to explicitly express their gratitude towards an addressee or a group of addressees, as shown in the above example, which contains two sentences, the first one indicating gratitude, addressee, and reason of thanking, and the second one elaborating on the assistance provided by the addressee. The two sentences, however, contain only one semantic category of thanking. It was then established that, for the counting of occurrences of explicit thanking strategies, when there is only one occurrence of thanking word(s) and one occurrence of addressee(s) in one or more sentences, an occurrence of explicit thanking strategy was counted. With this criterion, therefore, when two occurrences of thanking occur in two or more sentences that are concerned with only one addressee, two occurrences of explicit thanking strategies are counted. Following is an example:

2) I would like to express my sincere gratitude to my advisor, Dr. White, for all her guidance and support. I appreciate her continuous encouragement and advice during the writing of this thesis. Were it not for her help, the completion of the thesis would not have been possible ( $\mathrm{S} 2+\mathrm{C} 9$, advisor, TS2).

In the above example, the first sentence contains the semantic category of thanking, the category of addressee, and the category of reason (favor). The second sentence is concerned with the same addressee; however, it contains a different thanking word (that is, appreciate) and a different reason (favor and

Table 1.

The classification of explicit thanking strategies.

\begin{tabular}{|c|c|}
\hline \multicolumn{2}{|r|}{ Explicit Thanking Strategies } \\
\hline \multicolumn{2}{|r|}{ (i) Simple thanking: } \\
\hline S1 & [thanking] + [addressees] \\
\hline $\mathrm{S} 2$ & [thanking] + [addressees] + [reason (favor)] \\
\hline S3 & [thanking] + [addressees] + [reason (positive feelings) $]$ \\
\hline S4 & [thanking] + [addressees] + [indispensability] \\
\hline S5 & [thanking] + [addressees] + [reason (favor and positive feelings)] \\
\hline \multicolumn{2}{|r|}{ (ii) Complex thanking: } \\
\hline $\mathrm{C} 1$ & [thanking] + [addressees] + [reason (favor)] + [elaboration] \\
\hline $\mathrm{C} 2$ & [thanking] + [addressees] + [reason (positive feelings)] + [elaboration] \\
\hline С3 & [thanking] + [addressees] + [reason (favor)] + [indispensability $]$ \\
\hline $\mathrm{C} 4$ & [thanking] + [addressees] + [reason (positive feelings)] + [indispensability] \\
\hline C5 & [thanking] + [addressees] + [reason (favor)] + [elaboration] + [indispensability] \\
\hline C6 & [thanking] + [addressees] + [reason (positive feelings) $]+$ [elaboration] + [indispensability $]$ \\
\hline C7 & [thanking] + [addressees] + [indispensability] + [elaboration] \\
\hline $\mathrm{C} 8$ & [thanking] + [addressees] + [reason (favor \& positive feelings)] + [elaboration] \\
\hline C9 & [thanking] + [addressees] + [reason (favor \& positive feelings)] + [indispensability] \\
\hline C10 & [thanking] + [addressees] + [reason (favor \& positive feelings)] + [elaboration] $+[$ [indispensability] \\
\hline
\end{tabular}


positive feelings). The third sentence contains no thanking, but has a new category of indispensability. Therefore, the first sentence was identified as a thanking strategy of S2, while the second and the third sentences together were identified as a thanking strategy of C9.

For analysis of linguistic realizations, lexical items that explicitly express gratitude, such as thank, appreciate, gratitude, indebted, and grateful, were identified. A text analysis and concordance program AntConc was used to count and retrieve explicit thanking expressions from the corpus, but implicit thanking expressions were identified manually. A subset of the data were coded independently by a second rater to ensure the reliability of analysis.

\section{Results and Discussion}

\section{Thanking Strategies}

Analysis of the TS and NAS corpora reveals that in average, TS's thesis acknowledgements are longer than NAS's; however, the length variation is greater for NAS than TS, as indicated previously in section 2.1. This is consistent with the result shown in Table 2 that TS use more thanking strategies (265) than NAS (244). In addition, TS writers use much more explicit thanking strategies (252) than NAS writers (189), but in contrast, TS writers use much fewer implicit thanking strategies (13) than NAS writers (55), although in both groups explicit thanking strategies are used much more frequently than implicit thanking strategies. It seems that TS writers rely mainly on overt thanking words to express their gratitude, and they tend not to use covert expressions of thanks. It is suspected that this may be related to the academic convention or norm in the Taiwanese academic discourse community to express gratitude explicitly in acknowledgements so as to show the students' recognition of assistance from others in writing theses. In contrast, slightly less than one fourth of the strategies (22.5\%) by NAS are implicit. However, as I examined the occurrences of the implicit strategies in the NAS data, it was found that as a matter of fact, many of the implicit strategies concentrate in a few acknowledgements, suggesting that the result is probably influenced by individual writing styles. The examination of the implicit thanking strategies also revealed that in most cases the NAS writers emphasize the specific assistance they have received from various addressees. Nevertheless, as the TS use implicit thanking strategies, most of them indicate the importance or indispensability of the help or moral support provided by the addressees. In addition, these implicit strategies do not concentrate in a small number of acknowledgements. The following two examples illustrate the implicit thanking strategy used by a native student writer, where he indicates the assistance he receives from various people without using an overt thanking expression, and then the implicit thanking strategy by a nonnative student writer, where he indicates the indispensability of his friend's love and support.

Table 2.

Frequency of thanking strategies for TS and NAS.

\begin{tabular}{ccc}
\hline & TS (\%) & NAS (\%) \\
\hline Explicit thanking & $252(95.1)$ & $189(77.5)$ \\
Implicit thanking & $13(4.9)$ & $55(22.5)$ \\
Total & $265(100.0)$ & $244(100.0)$ \\
\hline
\end{tabular}

3) I have gained deep respect for the faculty on my committee at UX during my time in the MA TESOL program. My committee chair, Dr. Paul Baron has helped me develop my writing... Dr. Lynn Collins helped me to keep a sense of humor... Dr. Mary Smith Campbell helped me to see the bigger picture... (implicit, NAS12).

4) Finally, to Mary, without whom I would never have considered that this was possible. Her love and support motivated me on the occasions when things seemed out of reach (implicit, TS9).

Due to the large proportion of explicit thanking strategies used in both groups, the category was further classified into five simple thanking strategies and ten complex thanking strategies. Table 3 shows that simple thanking strategies outnumber complex thanking strategies in the two groups, with $68.7 \%$ in TS and $79.9 \%$ in NAS. The percentage of simple thanking strategies exceeds at least twice as high as that of complex thanking strategies in each group (around 2.2 times in the TS group and 4 times in the NAS group). Therefore, it seems both groups have a preference of simple thanking strategies over complex thanking strategies; however, NAS use more simple thanking strategies than TS, whereas TS use more complex thanking strategies than NAS. This result is somewhat unexpected in view of the linguistic resources of the two groups. However, from a socio-cultural perspective, it is possible that more TS than NAS may consider thesis acknowledgements as a very formal genre and thus the use of complex thanking strategies, which express the student writers' gratitude in a more elaborative way, are formal and appropriate. Two other factors may also contribute to this result; one is the socio-cultural expectation of expressing gratitude to the respectable or helpful persons like advisors and family; the other is the interpersonal relationship between students and addressees. In Taiwan, advisors or committee members are deemed authoritative and very helpful, usually guiding students closely throughout their thesis

Table 3.

Frequency of subcategories of explicit thanking strategies for TS and NAS.

\begin{tabular}{cccc}
\hline & TS (\%) & NAS (\%) & Total (\%) \\
\hline S1 & $15(6.0)$ & $9(4.8)$ & $24(5.4)$ \\
S2 & $92(36.5)$ & $84(44.4)$ & $176(39.9)$ \\
S3 & $42(16.7)$ & $42(22.2)$ & $84(19.1)$ \\
S4 & $8(3.2)$ & $2(1.1)$ & $10(2.3)$ \\
S5 & $16(6.3)$ & $14(7.4)$ & $30(6.8)$ \\
Subtotal & $173(68.7)$ & $151(79.9)$ & $324(73.5)$ \\
C1 & $20(7.9)$ & $17(9.0)$ & $37(8.4)$ \\
C2 & $22(8.7)$ & $8(4.2)$ & $30(6.8)$ \\
C3 & $11(4.4)$ & $4(2.1)$ & $15(3.4)$ \\
C4 & $5(2.0)$ & $1(0.5)$ & $6(1.4)$ \\
C5 & $5(2.0)$ & $0(0.0)$ & $5(1.1)$ \\
C6 & $2(0.8)$ & $0(0.0)$ & $2(0.5)$ \\
C7 & $2(0.8)$ & $0(0.0)$ & $2(0.5)$ \\
C8 & $5(2.0)$ & $6(3.2)$ & $11(2.5)$ \\
C9 & $4(1.6)$ & $2(1.1)$ & $6(1.4)$ \\
C10 & $3(1.2)$ & $0(0.0)$ & $3(0.7)$ \\
Subtotal & $79(31.4)$ & $38(20.1)$ & $117(36.5)$ \\
Total & $252(100.0)$ & $189(100.0)$ & $441(100.0)$ \\
\hline
\end{tabular}

Note: S1-5 = simple thanking strategies; C1-10 = complex thanking strategies. 
writing process, while in America or Canada, advisorship seems to emphasize the cultivation of students' independent ability of research. These differences in socio-cultural perceptions between the two groups may affect not only the kind of assistance advisors and faculty provide for students but also the interpersonal relationship between advisors and advisees, both of which may lead to the kind of expressions of gratitude that students perceive as appropriate in their thesis acknowledgements. Two other findings, as shown in Table 3, support this interpretation. First, it can be observed that TS use much more thanking strategies that contain the semantic category of indispensability (S4, C3, C4, C5, C6, C7, C9, and C10) than NAS, 40 and 9 occurrences, respectively. Also, four strategies that contain both the semantic category of elaboration and that of indispensability, that is, C5, C6, C7, and C10, are not used by NAS at all, while TS use all types of thanking strategies. In addition, S2 and S3 are the two most frequently used thanking strategies in the two groups, accounting for $53.2 \%$ in TS and $66.6 \%$ in NAS. This suggests that both TS and NAS are prone to express their gratitude towards addressees by simply noting the favor they receive (e.g., advice on thesis, or help with statistic analysis) or stating positive feelings (moral support and encouragement) they experience. This seems to be the conventional use of thanking expressions.

With regard to complex thanking strategies, where students not only express their thanks and reasons, but also elaborate on the addressees' specific assistance, C1 ([thanking] + [addressees] + [reason, favor] + [elaboration]) is most commonly used by NAS, whereas both C1 and C2 ([thanking] + [addressees] + [reason, positive feelings] + [elaboration]) are commonly used by TS, as also shown in Table 3 . It can also be noted that TS use much more C2 and C3 ([thanking] + [addressees] + [reason, favor] + [indispensability]) than NAS, reflecting that more TS than NAS tend to elaborate on the assistance from addressees and their indispensability.

To sum up, the quantitative analysis of acknowledgements in terms of thanking strategies generally reveals that TS are similar to NAS in that they use explicit strategies much more frequently than implicit strategies and employ simple strategies much more frequently than complex strategies. Nevertheless, the differences between the two groups in the relative percentages of the types of strategies or the high-frequency strategies, as indicated above, may reflect subtle divergence as a result of socio-cultural perceptions of expressing gratitude in thesis acknowledgements. While previous studies on acknowledgements have identified the rhetorical structure of thesis acknowledgements and gratitude expressions (Al-Ali, 2010; Hyland, 2004; Hyland \& Tse, 2004), analyzing the various types of thanking strategies and comparing the use of these strategies between NAS and TS writers have revealed how socio-cultural pragmatism of the student writers may clarify subtle distinction between these two groups in expressing thanking in MA thesis acknowledgements.

The differences between the two groups can be more interestingly and vividly illustrated by the linguistic realizations of the thanking strategies each group uses, the arrangement of addressees, and the relations between thanking strategies and addressees.

\section{Linguistic Relations of Thanking Strategies}

As indicated in the earlier section, explicit thanking strategies constitute a considerable percentage in both groups. There- fore, further investigation was conducted to examine how explicit thanking strategies are realized linguistically in terms of parts of speech and lexical realizations. Table 4 shows that, overall, nouns and verbs are mainly used to express gratitude in thesis acknowledgements. This is consistent with Hyland and Tse's (2004) finding. However, TS and NAS prefer different language forms to express thanks. TS prefer the noun form (55.2\%), while NAS use the verb form (47.5\%) more frequently. For example, thanks expressed through nominalization, such as "My gratitude goes to..." or "Special thanks go to..." are more heavily used by TS, whereas performative verbs, such as "I thank...” are more commonly used by NAS.

Further analysis of specific words used in explicit thanking strategies reveals, as shown in Table 5, that the word thank(s), irrespective of part of speech, is used most frequently than any other thanking words, accounting for $44.1 \%$ and $57 \%$ of all occurrences in the TS and the NAS data, respectively. Specifically, thank in the verb form occurs most frequently in both the TS text (23.6\%) and the NAS text (36.0\%), with a higher ratio in NAS. The top five thanking words in TS, in terms of their frequency, are thank, gratitude, thanks, appreciation, and grateful, while in NAS, the top five are thank, thanks, grateful, gratitude, and acknowledge. In other words, thank, gratitude, thanks, and grateful are the four overt thanking words that both TS and NAS prefer to use in theses acknowledgements. How-

Table 4.

Frequency of parts of speech in explicit thanking for TS and NAS.

\begin{tabular}{ccc}
\hline & TS (\%) & NAS (\%) \\
\hline Noun & $143(55.2)$ & $73(36.5)$ \\
Verb & $78(30.1)$ & $95(47.5)$ \\
Adjective & $38(14.7)$ & $32(16.0)$ \\
Total & $259(100.0)$ & $200(100.0)$ \\
\hline
\end{tabular}

Table 5.

Frequency of lexical realiztions of explicit thanking strategies for TS and NAS.

\begin{tabular}{cccc}
\hline & Lexical items & TS (\%) & NAS (\%) \\
\hline Noun & gratitude & $57(22.0)$ & $17(8.5)$ \\
& thanks & $53(20.5)$ & $42(21.0)$ \\
& appreciation & $25(9.7)$ & $7(3.5)$ \\
& gratefulness & $3(1.2)$ & $1(0.5)$ \\
& indebtedness & $2(0.8)$ & $1(0.5)$ \\
& debt & $3(1.2)$ & $3(1.2)$ \\
Verb & Thank-you & $0(0.0)$ & $1(0.5)$ \\
& recognition & $0(0.0)$ & $1(0.5)$ \\
& thank & $61(23.6)$ & $72(36.0)$ \\
& appreciate & $6(2.3)$ & $1(0.5)$ \\
& appreciated & $2(0.8)$ & $4(2.0)$ \\
& acknowledge & $5(1.9)$ & $10(5.0)$ \\
& recognize & $0(0.0)$ & $2(1.0)$ \\
& owe & $4(1.5)$ & $6(3.0)$ \\
Total & grateful & $19(7.3)$ & $22(11.0)$ \\
& indebted & $12(4.6)$ & $6(3.0)$ \\
& thankful & $5(1.9)$ & $2(1.0)$ \\
& appreciative & $2(0.8)$ & $1(0.5)$ \\
& obliged & $0(0.0)$ & $1(0.5)$ \\
& & $259(100.0)$ & $200(100.0)$ \\
\hline
\end{tabular}


ever, Table 5 also shows that some thanking words have a relatively higher frequency in one group than the other. For instance, gratitude and appreciation, both are nouns, occur much more frequently in TS than in NAS, while thank, grateful, and acknowledge, which are verbs or adjective, are used much more frequently by the NAS than by the TS. These findings once again corroborate the previous conclusion that TS tend to use more noun forms to express their thanks, whereas NAS employ more verb forms. Examination of the generic context of these thanking words or patterns that are often linked to these thanking words also yields some interesting findings. For example, for high-frequency nouns gratitude/appreciation/thanks, the use of a formulaic pattern, my (deepest/sincere/...) gratitude/ appreciation/thanks goes to has 27 occurrences in the TS data, but merely 3 occurrences in the NAS data. Another formulaic pattern is the use of a preface, as indicated in Hyland and Tse (2004). Hyland and Tse found that thesis acknowledgements in their corpus are likely to contain a preface such as I would like to..., used formulaically to add an element of formality. It was found that this fixed phrase is used 56 times in the TS acknowledgements, and 35 times in the NAS acknowledgements. These results seem to suggest that non-native English speaker master students (in this study, Taiwanese students) tend to construct their thanking expressions with more conventionalized set patterns than native English speaker master students (American or Canadian students), and these patterns are probably intended for increasing the formality of acknowledgements.

\section{Categories and Arrangements of Addressees}

One of the most crucial elements of thesis acknowledgements is the thanked addressees to whom students express their gratitude. The semantic category of addressees was further classified into seven subcategories: 1) advisors, referring to thesis advisors; 2) committee members, referring to those who serve on the thesis committee; 3 ) other faculty members, referring to faculty who are not on the thesis committee, such as faculty from students' departments or university, or from their previous schools or from other institutions or universities; 4) participants, helpers or institutions, referring to those who par- ticipate in the study, those who provide assistance for students to access the participants, or provide assistance of statistic analysis or research funding; 5) classmates or friends; 6) family members, including parents, grandparents, relatives, siblings, spouses, children, boyfriends and girlfriends; 7) others, referring to anyone or anything else that does not fit into the previous categories, such as pets, God or music.

Of all the seven subcategories of addressees, each TS acknowledgement generally contains 3 to 7 subcategories, whereas each NAS acknowledgement contains 1 to 6 categories, with a mean of 5.3 and 4.6 addressees, respectively. As shown in Table 6, all categories of addressees are frequently mentioned in MA thesis acknowledgements for both TS and NAS groups, except the category of others. The most thanked addressees are advisors, committee members and family members. These three categories occur in all TS acknowledgements; however, only $80.0 \%-83.3 \%$ of NAS thank all these addressees in their acknowledgements. The next frequent categories are other faculty members, participants/helpers/institutions, and classmates/friends, with a frequency ranging from $63.3 \%$ to $83.3 \%$. Interestingly, one native student writer thanks only one category of addressees in his acknowledgement, namely, participants/helpers/institutions. This somehow indicates the importance of participants and the difficulty of conducting research in applied linguistics, where assistance is always needed and highly appreciated. Three native student writers thank only two categories of addressees, two of whom thank advisors and committee members, and the other thanks friends and family members. Another finding worth noting is that there are only four occurrences for the category of others, including a cat, a dog, music and Jesus (see Examples 5 and 6). Example 5 comes from TS, and Example 6 from NAS.

5) I would also like to extend my sincere appreciation to music because music has always been and will be the backbone of my life: "In Music We Trust." (C2, TS29).

6) Words on a page seem inadequate to thank my Savior, Jesus for what He has done to supply the joy on this road of life. His provision was abundant in bringing so many amazing people to aide me in this specific chapter of the journey. (C2,

Table 6.

Arrangement order of thanked addressees for TS and NAS.

\begin{tabular}{|c|c|c|c|c|c|c|c|c|c|c|c|c|c|c|}
\hline \multirow[b]{2}{*}{ Order } & \multicolumn{2}{|c|}{$\mathrm{a}$} & \multicolumn{2}{|c|}{$\mathrm{b}$} & \multicolumn{2}{|c|}{ c } & \multicolumn{2}{|c|}{$d$} & \multicolumn{2}{|c|}{ e } & \multicolumn{2}{|c|}{$\mathrm{f}$} & \multicolumn{2}{|c|}{ g } \\
\hline & TS & NAS & TS & NAS & TS & NAS & TS & NAS & TS & NAS & TS & NAS & TS & NAS \\
\hline 1 & 30 & 17 & 0 & 1 & 0 & 2 & 0 & 5 & 0 & 2 & 0 & 3 & 0 & 0 \\
\hline 2 & 0 & 2 & 27 & 18 & 1 & 3 & 0 & 1 & 0 & 2 & 2 & 3 & 0 & 0 \\
\hline 3 & 0 & 4 & 2 & 2 & 12 & 6 & 7 & 7 & 7 & 4 & 2 & 1 & 0 & 0 \\
\hline 4 & 0 & 2 & 0 & 2 & 4 & 6 & 10 & 4 & 8 & 2 & 7 & 4 & 0 & 0 \\
\hline 5 & 0 & 0 & 0 & 2 & 1 & 1 & 3 & 2 & 5 & 7 & 7 & 2 & 1 & 0 \\
\hline 6 & 0 & 0 & 1 & 0 & 0 & 1 & 0 & 1 & 3 & 1 & 4 & 6 & 1 & 0 \\
\hline 7 & 0 & 0 & 0 & 0 & 2 & 0 & 0 & 0 & 1 & 2 & 4 & 2 & 0 & 1 \\
\hline 8 & 0 & 0 & 0 & 0 & 0 & 0 & 0 & 0 & 1 & 1 & 3 & 2 & 0 & 0 \\
\hline 9 & 0 & 0 & 0 & 0 & 0 & 0 & 0 & 0 & 0 & 0 & 1 & 1 & 1 & 0 \\
\hline Total & 30 & 25 & 30 & 25 & 20 & 19 & 20 & 20 & 25 & 21 & 30 & 24 & 3 & 1 \\
\hline Total (\%) & 100.0 & 83.3 & 100.0 & 83.3 & 66.7 & 63.3 & 66.7 & 66.7 & 83.3 & 70.0 & 100 & 80.0 & 10.0 & 3.3 \\
\hline Not mentioned & 0 & 5 & 0 & 5 & 10 & 11 & 10 & 10 & 5 & 9 & 0 & 6 & 27 & 29 \\
\hline
\end{tabular}

Note: $\mathrm{a}=$ advisor; $\mathrm{b}=$ committee members; $\mathrm{c}=$ other faculty members; $\mathrm{d}$ = participants, helpers or institutions; $\mathrm{e}=$ classmates or friends; $\mathrm{f}=\mathrm{family}$ members; $\mathrm{g}=$ others. $\mathrm{N}=30$ in TS; $\mathrm{N}=30$ in NAS. 
NAS12).

Therefore, people that generously offer assistance or support to TS or NAS are largely similar in terms of their role relationships with the students. A discipline-specific addressee is participants who serve as the sources of research data in applied linguistics and hence critical to the students' thesis research.

Table 6 also shows the arrangement order of addressees, indicating that advisors are always thanked first by TS, but not always so by NAS (17 out of 25 acknowledgements that thank advisors). Those who do not first thank advisors thank committee members, other faculty members, or participants/helpers/ institutions first. Similarly, committee members are more frequently thanked second by TS than NAS. Other categories of addressees, such as other faculty members, participants/helpers/ institutions, and classmates/friends, are most frequently mentioned in the mid position of the acknowledgements by both groups. As for the category of family members, it seems they can occur in any position of the acknowledgements, although they tend to occur towards the end. This, however, does not suggest that family members are not important to the student writers. Rather, the help provided by family members is usually moral support (as it will be later revealed that S3 is the most frequently used thanking strategy for family members) and in a practical sense may not be so directly related to thesis research. As a matter of fact, either the first or the last position can be a place to express the deepest gratitude to the most important person. Following are examples of family members thanked in the first and the last position, respectively:

7) Finally, my inexpressible debt is to my dearest family, in particular, my husband and my unborn baby, who is coming in around two or three weeks. My husband's constant support and understanding has made it possible for me to... (C2, TS10).

8) First of all, I wish to thank my husband Richard, who, I think, was even more excited than I when I made the decision to get this degree. He has been my chief cheering section and... (C2, NAS5).

To sum up, student writers' preference in ordering addresses, as reflected in the results of analysis, characterizes their social and cultural pragmatism. The TS are prone to arrange the thanked addressees in a more strict order; for example, they always thank advisors at the initial position of acknowledgements, usually followed by committee members, and often thank their family members at the end position of acknowledgements. Comparatively, only in a little more than half of the NAS acknowledgements, advisors are put in the first position, followed by committee members in about the same amount of acknowledgements, and family members are frequently put in later positions. These results show that TS and NAS have different perception of the role of advisors or the advisor-advisee relationship. In eastern academic culture (e.g., Chinese, Korean or Japanese), the hierarchical advisor-advisee relationships are unduly underscored (Krase, 2007; Li, 2005). Advisors, among a number of thanked addressees, are regarded as the most authoritative and powerful figures by their advisees since not only are they knowledgeable in the field, but they also play a critical role in deciding the pass or fail of student writers' theses. To emphasize their utmost importance, TS view advisors as indispensable addressees and always place them at the initial posi tion of acknowledgements.

Comparatively, in the egalitarian advisor-advisee relationship in the western academic culture, advisors are not considered the foremost authority but joint partners boosting mutual growth and enhancement with advisees (Krase, 2007). This, therefore, accounts for the more flexible arrangement of advisors by NAS. Similar result can be observed for the category of committee members and other faculty members.

Although both the beginning and the end positions can be reserved for the most essential and significant addressees, for most students, it seems a convention that the arrangement of addressees should be based on how the assistance provided by specific addressees is related to thesis writing. Advisors often provide most intellectual guidance to student writers whereas family members generally provide great moral support for them. Therefore, in either TS or NAS acknowledgements, advisors are usually thanked first, while family members are often thanked last, even though family members are utmost important to the students. Comparing TS and NAS arrangement of addressees reveals that NAS show more variation in arranging the thanked addressees; for example, all categories of addressees may occur in the first position of NAS acknowledgements, while only advisors occur in that position in TS acknowledgements.

\section{Relations between Thanking Strategies and Addressees}

In general, the categories of advisors, committee members, and other faculty members are thanked mostly for intellectual influence and guidance because they provide academic assistance to student writers in their process of writing theses. Some advisors are acknowledged for both academic guidance and moral support. Conversely, the categories of classmates/friends and family members, are mostly thanked for their moral support, such as encouragement, patience and care. Three TS and two NAS thank their family members not only for moral support but for financial support as well. In addition, most of the TS and NAS refer to people by their full names (i.e. first name + last name). In the case of thanking advisors, committee, or other faculty members, most student writers include titles (e.g., Dr. or Professor) followed by the full names of the professors.

Tables 7 and 8 show different thanking strategies for various addressees by TS and NAS, respectively. The results indicate that overall, for both groups, simple thanking strategies are used far more commonly than complex thanking strategies for most addressees except for the category of advisors in the TS data. TS tend to elaborate and expand on what their advisors have done for them. They (72.7\%) use much more complex thanking strategies than NAS (37.5\%); in contrast, NAS (50.0\%) use more simple thanking strategies than TS (24.2\%) for advisors. This not only suggests the critical role advisors play in the TS' process of thesis writing but the high gratitude and respect these students consider socially and academically appropriate to express in their thesis acknowledgements. It is also worth noting that these student writers use more than one strategy, or combine different strategies to thank advisors. The following example illustrates this phenomenon:

9) I would like to express my profound gratitude to my thesis advisor, Dr. Li-Yu Chen, who has made every effort possible to help me complete the thesis. I deeply appreciate her patience of reading each chapter over and over again and giving me valuable suggestions continuously for my thesis. Without her encouragement and guidance, my thesis would not be completed within one year (S2 + C3, advisor, TS12). 
Table 7.

Frequency of types of thanking strategies to addressees for TS.

\begin{tabular}{cccccccc}
\hline & $\mathrm{a}$ & $\mathrm{b}$ & $\mathrm{C}$ & $\mathrm{d}$ & $\mathrm{e}$ & $\mathrm{f}$ & $\mathrm{g}$ \\
\hline S1 & $0(0.0)^{\mathrm{a}}$ & $1(4.0)$ & $1(3.7)$ & $3(16.7)$ & $4(5.3)$ & $1(2.5)$ & $0(0.0)$ \\
S2 & $3(9.1)$ & $14(56.0)$ & $14(51.9)$ & $10(55.6)$ & $27(35.5)$ & $4(10.0)$ & $0(0.0)$ \\
S3 & $0(0.0)$ & $1(4.0)$ & $1(3.7)$ & $0(0.0)$ & $20(26.3)$ & $14(35.0)$ & $2(66.7)$ \\
S4 & $2(6.1)$ & $1(4.0)$ & $2(7.4)$ & $1(5.6)$ & $2(2.6)$ & $3(7.5)$ & $0(0.0)$ \\
S5 & $3(9.1)$ & $0(0.0)$ & $4(14.8)$ & $0(0.0)$ & $4(5.3)$ & $1(2.5)$ & $0(0.0)$ \\
Subtotal & $8(24.2)$ & $17(68.0)$ & $22(81.5)$ & $14(77.8)$ & $57(75.0)$ & $23(57.5)$ & $2(66.7)$ \\
C1 & $7(21.2)$ & $4(16.0)$ & $2(7.4)$ & $1(5.6)$ & $2(2.6)$ & $1(2.5)$ & $0(0.0)$ \\
C2 & $1(3.0)$ & $1(4.0)$ & $0(0.0)$ & $0(0.0)$ & $7(9.2)$ & $8(20.0)$ & $0(0.0)$ \\
C3 & $2(6.1)$ & $1(4.0)$ & $2(7.4)$ & $2(11.1)$ & $4(5.3)$ & $0(0.0)$ & $0(0.0)$ \\
C4 & $1(3.0)$ & $0(0.0)$ & $0(0.0)$ & $0(0.0)$ & $1(1.3)$ & $3(7.5)$ & $1(33.3)$ \\
C5 & $4(12.1)$ & $0(0.0)$ & $0(0.0)$ & $0(0.0)$ & $0(0.0)$ & $0(0.0)$ & $0(0.0)$ \\
C6 & $0(0.0)$ & $0(0.0)$ & $0(0.0)$ & $0(0.0)$ & $0(0.0)$ & $2(5.0)$ & $0(0.0)$ \\
C7 & $0(0.0)$ & $0(0.0)$ & $0(0.0)$ & $0(0.0)$ & $0(0.0)$ & $2(5.0)$ & $0(0.0)$ \\
C8 & $4(12.1)$ & $0(0.0)$ & $0(0.0)$ & $0(0.0)$ & $1(1.3)$ & $0(0.0)$ & $0(0.0)$ \\
C9 & $2(6.1)$ & $0(0.0)$ & $0(0.0)$ & $0(0.0)$ & $1(1.3)$ & $0(0.0)$ & $0(0.0)$ \\
C10 & $3(9.1)$ & $0(0.0)$ & $0(0.0)$ & $0(0.0)$ & $0(0.0)$ & $0(0.0)$ & $0(0.0)$ \\
Subtotal & $24(72.7)$ & $6(24.0)$ & $4(14.8)$ & $3(16.7)$ & $16(21.1)$ & $16(40.0)$ & $1(33.3)$ \\
I & $1(3.0)$ & $2(8.0)$ & $1(3.7)$ & $1(5.6)$ & $3(3.9)$ & $3(7.5)$ & $0(0.0)$ \\
Total & $33(100.0)$ & $25(100.0)$ & $27(100.0)$ & $18(100.0)$ & $76(100.0)$ & $40(100.0)$ & $3(100.0)$ \\
\hline
\end{tabular}

Note: $\mathrm{a}$ = advisor; $\mathrm{b}=$ committee members; $\mathrm{c}=$ other faculty members; $\mathrm{d}$ = participants, helpers or institutions; $\mathrm{e}=\mathrm{classmates}$ or friends; $\mathrm{f}=\mathrm{family}$ members; $\mathrm{g}=$ others;

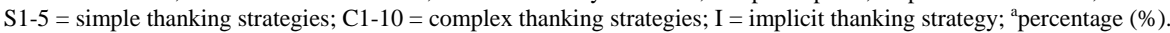

Table 8.

Frequency of types of thanking strategies to addressees for NAS.

\begin{tabular}{cccccccc}
\hline & $\mathrm{a}$ & $\mathrm{b}$ & $\mathrm{c}$ & $\mathrm{d}$ & $\mathrm{e}$ & $\mathrm{f}$ & $\mathrm{g}$ \\
\hline S1 & $2(8.3)^{\mathrm{a}}$ & $1(3.8)$ & $0(0.0)$ & $0(0.0)$ & $3(4.3)$ & $1(2.4)$ & $0(0.0)$ \\
S2 & $7(29.2)$ & $14(53.8)$ & $14(45.2)$ & $12(60.0)$ & $21(30.0)$ & $3(7.3)$ & $0(0.0)$ \\
S3 & $2(8.3)$ & $2(7.7)$ & $3(9.7)$ & $1(5.0)$ & $17(24.3)$ & $14(34.1)$ & $0(0.0)$ \\
S4 & $0(0.0)$ & $0(0.0)$ & $0(0.0)$ & $0(0.0)$ & $0(0.0)$ & $0(0.0)$ & $0(0.0)$ \\
S5 & $1(4.2)$ & $0(0.0)$ & $2(6.5)$ & $0(0.0)$ & $5(7.1)$ & $4(9.8)$ & $0(0.0)$ \\
Subtotal & $12(50.0)$ & $17(65.4)$ & $19(61.3)$ & $13(65.0)$ & $46(65.7)$ & $22(53.7)$ & $0(0.0)$ \\
C1 & $6(25.0)$ & $1(3.8)$ & $5(16.1)$ & $1(5.0)$ & $2(2.9)$ & $3(7.3)$ & $0(0.0)$ \\
C2 & $0(0.0)$ & $0(0.0)$ & $0(0.0)$ & $0(0.0)$ & $1(1.4)$ & $1(2.4)$ & $1(100.0)$ \\
C3 & $0(0.0)$ & $0(0.0)$ & $1(3.2)$ & $2(10.0)$ & $2(2.9)$ & $1(2.4)$ & $0(0.0)$ \\
C4 & $0(0.0)$ & $0(0.0)$ & $0(0.0)$ & $0(0.0)$ & $1(1.4)$ & $0(0.0)$ & $0(0.0)$ \\
C5 & $0(0.0)$ & $0(0.0)$ & $0(0.0)$ & $0(0.0)$ & $0(0.0)$ & $0(0.0)$ & $0(0.0)$ \\
C6 & $0(0.0)$ & $0(0.0)$ & $0(0.0)$ & $0(0.0)$ & $0(0.0)$ & $0(0.0)$ & $0(0.0)$ \\
C7 & $0(0.0)$ & $0(0.0)$ & $0(0.0)$ & $0(0.0)$ & $0(0.0)$ & $0(0.0)$ & $0(0.0)$ \\
C8 & $2(8.3)$ & $1(3.8)$ & $2(6.5)$ & $0(0.0)$ & $1(1.4)$ & $1(2.4)$ & $0(0.0)$ \\
C9 & $1(4.2)$ & $0(0.0)$ & $0(0.0)$ & $0(0.0)$ & $0(0.0)$ & $0(0.0)$ & $0(0.0)$ \\
C10 & $0(0.0)$ & $0(0.0)$ & $0(0.0)$ & $0(0.0)$ & $0(0.0)$ & $0(0.0)$ & $0(0.0)$ \\
Subtotal & $9(37.5)$ & $2(7.7)$ & $8(25.8)$ & $3(15.0)$ & $7(10.0)$ & $6(14.6)$ & $1(100.0)$ \\
I & $3(12.5)$ & $7(26.9)$ & $4(12.9)$ & $4(20.0)$ & $17(24.3)$ & $13(31.7)$ & $0(0.0)$ \\
Total & $24(100.0)$ & $26(100.0)$ & $31(100.0)$ & $20(100.0)$ & $70(100.0)$ & $41(100.0)$ & $1(100.0)$ \\
\hline
\end{tabular}

Note: $\mathrm{a}=$ advisor; $\mathrm{b}=$ committee members; $\mathrm{c}=$ other faculty members; $\mathrm{d}$ = participants, helpers or institutions; $\mathrm{e}=$ classmates or friends; $\mathrm{f}=$ family members; $\mathrm{g}=$ others;

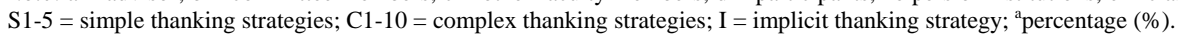

Therefore, one of the possible reasons that NAS use a higher frequency of simple thanking strategies for advisors is their preference of using two simple thanking strategies rather than one complex thanking strategy or the combination of one simple and one complex thanking strategy, which is preferred by TS. A combination of two simple thanking strategies can be observed in the following example from the NAS data:
10) To start, I would like to thank my mentor and the supervisor of this thesis, Dr. Janet Karlson. I am extremely appreciative of her constant support and encouragement over the past two years, both personal and academic (S1 + S5, advisor, NAS13).

Further examination of specific types of thanking strategies for various addressees reveals that S2 (indicating reasons due to 
the favor received) is most frequently used by both groups for four categories of addressees: committee members, other faculty members, participants/institutions, and classmates/friends, ranging from $30.0 \%$ to $60.0 \%$. However, for advisors, C1 (indicating reasons due to the favor received with further elaboration) is most frequently used by TS, while both S2 and C1 are more frequently used than other strategies by NAS. The most complicated thanking strategy, namely, C10 ([thanking] + [addressees] + [reason (favor \& positive feelings)] + [elaboration] + [indispensability]), is used in three TS acknowledgements, all for thanking advisors, while no occurrence of this strategy was found in the NAS acknowledgements. In terms of complex thanking strategies, NAS use only C1, C8 and C9 for advisors. One instance of C10 from the TS data is presented below:

11) My deepest gratitude is extended to my advisor, Dr. MeiLing Lin who has always been a motivator, an instructor, and a good friend throughout my Master research. She motivated me in times of despair, guided me in times of confusion, and offered guidance in times of chaos. Without her support and enlightenment, it would not have been possible for me to complete this interlanguage study, especially when the analysis of the results and the construction of the ideas baffled me (C10, advisor, TS19).

When thanking family members, both groups use S3 most frequently (35.0\% for TS and $34.1 \%$ for NAS), where positive feelings and moral support are indicated and highly valued. However, TS also tend to elaborate on the positive feelings, that is, using C2, whereas S5 is more often used by NAS, indicating both favor and positive feelings. For the addressee category of others, since in total there are only four occurrences of strategies, this category is not taken into account here.

The choice of strategies for different addressees often reflects different cultural perceptions of gratitude as well as the weight of addressees to TS and NAS. The use of thanking strategies may vary depending upon how students perceive the nature and the importance of the assistance or support the addressees have provided. For example, for both TS and NAS, S3, which focuses on positive feelings (usually moral support), is widely used for family members, while S2, which emphasizes favor (usually assistance related to thesis research), occurs most often for committee members. However, NAS use S2 for advisors most often, while TS most frequently employ $\mathrm{C} 1$; in other words, TS are more likely to not only indicate reasons, but also elaborate in detail on specific assistance that their advisors have done for them, while NAS tend to simply point out the assistance without much elaboration.

In conclusion, the subtle differences between TS and NAS in the use of thanking strategies, lexical choices, arrangements of addressees, and the relations between thanking strategies and addressees show that socio-cultural values and norms can shape the ways students express their thanks and influence the rhetorical structure of thesis acknowledgements, which reflect not only general academic conventions but the students' embedded socio-pragmatic perceptions of writing this genre as well.

\section{Conclusion}

As Hyland (2003: p. 265) indicates, “acknowledgements are sophisticated and complex textual constructs which bridge the personal and the public, the social and the professional, and the academic and the lay," the present study signifies thesis acknowledgements as the socio-pragmatic embodiment of the graduates' professional and interpersonal supporting networks. Thesis acknowledgements allow student writers to express publicly their personal gratitude; however, the way gratitude is expressed is mediated by the students' socio-cultural perceptions of generic appropriacy, as analyzed and exemplified in the acknowledgements in this study.

The pedagogical implications of this study are two-fold. Firstly, compared with other rhetorical sections in theses, acknowledgements have received much less, if any, attention in academic writing textbooks and style manuals. Hyland (2004) indicates that student writers often receive little instruction on the acknowledgement section, and they often look at other theses or dissertations to get ideas for structure, expression and content. Since acknowledgements come at the beginning of a thesis, a poor first impression may be made if they are inappropriately written (Swales \& Feak, 2000, 2004). How to write socio-culturally appropriate thesis acknowledgements is important for both NAS and TS.

Secondly, the contrastive analysis of acknowledgements in this study has unveiled interesting cross-cultural differences between TS and NAS not only in the use of thanking strategies but also in the arrangement preference for the thanked addressees. Graduate students should be explicitly informed of these discrepancies so as to meet various social norms and expectations.

More research remains to be done in order to create a general model of acknowledgements for students, instructors, as well as textbook writers. This study emphasizes the comparison of master thesis acknowledgements by Chinese native speakers and English native speakers in the field of applied linguistics. Further research can expand to include $\mathrm{PhD}$ dissertations, more samples of acknowledgements, native speakers of other languages, or other disciplines. In addition, most studies have focused on the perspectives of the student writers, but none has focused on the perspectives of the thanked addressees, such as advisors. Questions such as how advisors perceive acknowledgements or what kinds of acknowledgements they perceive as appropriate and what not can be further investigated. In particular, a pragmatic discourse analysis approach to these issues will help us better understand the complex socio-pragmatic interactions in acknowledgements.

\section{REFERENCES}

Al-Ali, M. N. (2010). Generic patterns and socio-cultural resources in acknowledgements accompanying Arabic Ph.D. dissertations. Pragmatics, 20, 1-26.

Bach, K., \& Harnish, R. M. (1979). Linguistic communication and speech acts. Cambridge: The MIT Press.

Bazerman, C. (1984). Modern evolution of the experimental report in physics: Spectroscopic articles in Physical Review, 1893-1980. Social Studies of Science, 14, 163-196. doi:10.1177/030631284014002001

Bazerman, C. (1988). Shaping written knowledge. Madison: University of Wisconsin Press.

Cronin, B. (1991). Let the credits roll: A preliminary examination of the role played by mentors and trusted assessors in disciplinary formation. Journal of Documentation, 47, 227-239. doi:10.1108/eb026878

Cronin, B. (1995). The Scholar's courtesy. The role of acknowledgement in the primary communication process. London: Taylor Graham.

Cronin, B., Mckenzie, G., \& Rubio, L. (1993). The norms of acknowledgement in four humanities and social sciences disciplines. Journal 
of Documentation, 49, 29-43. doi:10.1108/eb026909

Cronin, B., Mckenzie, G., \& Stiffler, M. (1992). Patterns of acknowledgement. Journal of Documentation, 48, 107-122. doi:10.1108/eb026893

Cronin, B., \& Overfelt, K. (1994). The scholar's courtesy: A survey of acknowledgement behavior. Journal of Documentation, 50, 164-196. doi:10.1108/eb026929

Giannoni, D. S. (2002). Worlds of gratitude: A contrastive study of acknowledgement texts in English and Italian research articles. Applied Linguistics, 23, 1-31. doi:10.1093/applin/23.1.1

Hyland, K. (2003). Dissertation acknowledgements: The anatomy of a Cinderella genre. Written Communication, 20, 242-268.

Hyland, K. (2004). Graduates' gratitude: The generic structure of dissertation acknowledgements. English for Specific Purposes, 23, 303-324. doi:10.1016/S0889-4906(03)00051-6

Hyland, K., \& Tse, P. (2004). "I would like thank my supervisor": Acknowledgements in graduate dissertations. International Journal of Applied Linguistics, 14, 259-275. doi:10.1111/j.1473-4192.2004.00062.x

Krase, E. (2007). "Maybe the communication beteen us was not enough": Inside a dysfunctional advisor/L2 advisee relationship. Journal of English for Academic Purposes, 6, 55-70. doi:10.1016/j.jeap.2006.12.001

$\mathrm{Li}$, Y. (2005). Multidimensional enculturation: The case of an EFL Chinese doctoral student. Journal of Asian Pacific Communication, 15, 153-170. doi:10.1075/japc.15.1.10li

Searle, J. R. (1969). Speech acts: An essay in the philosophy of language. Cambridge: Cambridge University Press.

Swales, J. M., \& Feak, C. B. (2000). English in today's research world: A writing guide. Ann Arbor, MI: The University of Michigan Press.

Swales, J. M., \& Feak, C. B. (2004). Academic writing for graduate students: Essential tasks and skills (2nd ed.). Ann Arbor, MI: The University of Michigan Press. 